www.nature.com/clinicalpractice/onc

\title{
Why perform sentinel-lymph-node biopsy in patients with melanoma?
}

\author{
Steven A Rosenberg
}

Sentinel-lymph-node biopsy (SLNB) has become the standard of care for patients presenting with intermediate-thickness melanoma; however, there is no evidence that this procedure has a positive impact on survival. One study (Morton DL [2006] N Engl J Med 355: 1307-1317) was elegantly designed to determine the impact of SLNB on patient survival. In total, 1,269 patients with melanoma and no clinically palpable lymph nodes were prospectively randomized to receive either observation or SLNB, which if positive, was followed by lymph-node dissection. There was no suggestion of a difference in survival when the two groups were compared $(P=0.58)$.

Many subgroup analyses were performed on these data that had little bearing on this main point. The survival analysis comparing patients with positive sentinel-lymph nodes to patients who recurred with nodal disease is difficult to interpret since it assumes no false-positive analyses of the sentinel nodes and that all abnormal cells in the nodes would eventually form a tumor. This is not a valid assumption as discussed by Thomas in this issue of the journal (Thomas M [2008] Nat Clin Pract Oncol 5: 18-23). Whether one believes the assumption is correct or not, it does not alter the compelling data that there
If the authors

conclude

that SLNB is

"preferred"

despite finding

no survival

benefit

associated

with this

procedure, one

wonders why

the study was

done at all.

SA Rosenberg is an Advisory Board member of Nature Clinical Practice Oncology.

\section{Competing interests}

The author declared no competing interests.

www.nature.com/clinicalpractice doi:10.1038/ncponc1022 was no survival difference between the two groups. This finding is in accordance with several prospective, randomized trials, all of which showed that there was no survival benefit to prophylactic lymph-node dissection in patients with melanoma. (Veronesi U et al. [1977] N Engl J Med 297: 627-630; Sim FH et al. [1978] Cancer 41: 948-956)

The small difference in progression-free survival that was observed in the Morton study was most likely attributable to the surgical removal of the nodes in one group, which removed the most common first site of recurrence in one group but not in the other.

The concluding sentence of the paper describing the randomized trial states that "...sentinel-lymph node biopsy should be preferred to observation". This conclusion is not justified by the data and should not be accepted. Although SLNB does provide prognostic information, patients should be clearly informed that SLNB, possibly followed by lymph-node dissection, is an expensive procedure with associated morbidity that confers no survival benefit. If the authors conclude that SLNB is "preferred" despite finding no survival benefit associated with this procedure, one wonders why the study was done at all. 